\title{
A Prospective Study on Red Blood Cell Transfusion Related Hyperkalemia in Critically III Patients
}

\author{
Shahzad Raza, b, d, Mahadi Ali Baig ${ }^{\mathrm{c}}$, Christopher Chang ${ }^{\mathrm{a}}$, Ridhima Dabas ${ }^{\mathrm{a}}$, Mallika Akhtar ${ }^{\mathrm{a}}$, Areej Khan ${ }^{\mathrm{a}}$, \\ Krishna Nemani ${ }^{\mathrm{a}}$, Rahima Alani ${ }^{\mathrm{a}}$, Omran Majumder ${ }^{\mathrm{a}}$, Natalya Gazizova ${ }^{\mathrm{a}}$, Shaluk Biswas ${ }^{\mathrm{a}}$, \\ Priyeshkumar Patel ${ }^{\mathrm{a}}$, Jaffar A. Al-Hillia ${ }^{\mathrm{a}}$, Yasar Shad ${ }^{\mathrm{a}}$, Barbara J. Berger ${ }^{\mathrm{a}}$, Mohammad Zaman ${ }^{\mathrm{a}}$
}

\begin{abstract}
Background: Transfusion-associated hyperkalemic cardiac arrest is a serious complication in patients receiving packed red blood cell (PRBC) transfusions. Mortality from hyperkalemia increases with large volumes of PRBC transfusion, increased rate of transfusion, and the use of stored PRBCs. Theoretically, hyperkalemia may be complicated by low cardiac output, acidosis, hyperglycemia, hypocalcemia, and hypothermia. In this study, we focus on transfusion-related hyperkalemia involving only medical intensive care unit (MICU) patients.

Method: This prospective observational study focuses on PRBC transfusions among MICU patients greater than 18 years of age. Factors considered during each transfusion included patient's diagnosis, indication for transfusion, medical co-morbidities, acid-base disorders, $\mathrm{K}^{+}$levels before and after each PRBC transfusion, age of stored blood, volume and rate of transfusion, and other adverse events. We used Pearson correlation and multivariate analysis for each factor listed above and performed a logistic regression analysis.
\end{abstract}

Results: Between June 2011 and December 2011, 125 patients received a total of 160 units of PRBCs. Median age was 63 years (22 - 92 years). Seventy-one (57\%) were females. Sixty-three patients $(50 \%)$ had metabolic acidosis, $75(60 \%)$ had acute renal failure $(\mathrm{ARF})$, and $12(10 \%)$ had end-stage renal disease (ESRD). Indications for transfusion included septic shock $(\mathrm{n}=65,52 \%)$, acute blood loss $(\mathrm{n}=25,20 \%)$, non-ST elevation myocardial infarction (NSTEMI) ( $\mathrm{n}$ $=25,20 \%)$ and preparation for procedures $(\mathrm{n}=14,11 \%)$. Baseline $\mathrm{K}^{+}$value was $3.9 \pm 1.1 \mathrm{mEq} / \mathrm{L}$ compared to $4.3 \pm 1.2 \mathrm{mEq} / \mathrm{L}$ posttransfusion respectively $(\mathrm{P}=0.9)$. During this study period, $4 \%$ of patients developed hyperkalemia $\left(\mathrm{K}^{+} 5.5 \mathrm{mEq} / \mathrm{L}\right.$ or above). The mean

Manuscript accepted for publication March 11, 2015

aDepartment of Internal Medicine, Brookdale University Hospital \& Medical Center, 1 Brookdale Plaza, Brooklyn, New York, NY 11212, USA

bUniversity of Missouri Columbia, Ellis Fischel Cancer Center, Columbia, Missouri, MO 65212, USA

${ }^{c}$ Albert Einstein School of Medicine, Montefiore Medical Center, 111 East 210th Street, Bronx, NY 10467, USA

${ }^{\mathrm{d} C o r r e s p o n d i n g ~ A u t h o r: ~ S h a h z a d ~ R a z a, ~ D e p a r t m e n t ~ o f ~ I n t e r n a l ~ M e d i c i n e, ~}$ Brookdale University Hospital \& Medical Center, 1 Brookdale Plaza, Brooklyn, New York, NY 11212, USA. Email: razashahzad2@gmail.com

doi: http://dx.doi.org/10.14740/jocmr2123w change of serum potassium in patients receiving transfusion $\geq 12$ days old blood was $4.1 \pm 0.4 \mathrm{mEq} / \mathrm{L}$ compared to $4.8 \pm 0.3 \mathrm{mEq} / \mathrm{L}$ (mean \pm SD) in patients receiving blood 12 days or less old. Sixty-two patients (77.5\%) that were transfused stored blood (for more than 12 days) had increased serum $\mathrm{K}^{+}$; eight $(17.7 \%)$ patients received blood that was stored for less than 12 days. In both univariate $(\mathrm{P}=0.02)$ and multivariate $(\mathrm{P}=0.04)$ analysis, findings showed that among all factors, transfusion of stored blood was the only factor that affected serum potassium levels (95\% CI: 0.32 - 0.91). No difference was found between central and peripheral intravenous access $(\mathrm{P}=0.12)$, acidosis $(\mathrm{P}$ $=0.12), \operatorname{ARF}(\mathrm{P}=0.6), \mathrm{ESRD}(\mathrm{P}=0.5)$, and multiple transfusions $(\mathrm{P}$ $=0.09)$. One subject developed a sustained cardiac arrest after developing severe hyperkalemia $\left(\mathrm{K}^{+}=9.0\right)$ following transfusion of seven units of PRBCs. Multivariate logistic regression showed linear correlation between duration of stored blood and serum $\mathrm{K}^{+}\left(\mathrm{R}^{2}=0.889\right)$.

Conclusion: This study assesses factors that affect $\mathrm{K}^{+}$in patients admitted to MICU. Results from the study show that rise in serum $\mathrm{K}^{+}$level is more pronounced in patients who receive stored blood ( $>12$ days). Future studies should focus on the use of altered storage solution, inclusion of potassium absorption filters during transfusion and cautious use of blood warmer in patients requiring massive blood transfusions.

Keywords: Hyperkalemia; Red cell storage; Packed red blood cell transfusion; Potassium

\section{Introduction}

Transfusion of stored red blood cells (RBCs) is associated with a wide range of complications that include circulatory overload, bradykinin-mediated hypotension, allergic reactions, coagulopathies, acute lung injury, infections, and death [1]. Hyperkalemia is a common complication in transfusion of stored blood. The supernatant of stored RBCs usually contains more than $60 \mathrm{mEq} / \mathrm{L}$ of potassium [2]. Potassium in stored blood increases due to decrease in ATP production and leakage of potassium into the supernatant. The initial high levels of potassium in stored blood predispose to post-transfusion hyperkalemia.

Cardiac arrest has been commonly reported in transfusionassociated hyperkalemia. Most cardiac arrests were reported in children and adult patients requiring massive blood transfusions $[3,4]$. Hypokalemia was rarely reported as a transfusion- 
Table 1. Patient Characteristics

\begin{tabular}{|c|c|c|}
\hline Gender & $\mathrm{N}=125$ & $\%$ \\
\hline Female & 71 & 56 \\
\hline Male & 54 & 43.2 \\
\hline Age & 63 (median) & 22 - 92 years \\
\hline \multicolumn{3}{|l|}{ Indication for PRBC transfusion } \\
\hline Acute blood loss & 25 & 16.6 \\
\hline Coronary artery disease with NSTEMI & 25 & 16.6 \\
\hline Septic shock & 61 & 49 \\
\hline Invasive procedure & 14 & 11.2 \\
\hline \multicolumn{3}{|l|}{ No. of PRBC transfusions } \\
\hline 1 unit & 104 & 83.2 \\
\hline 2 units & 11 & 8.8 \\
\hline 3 units & 9 & 7.2 \\
\hline 7 units & 1 & 0.8 \\
\hline \multicolumn{3}{|l|}{ Intravenous access } \\
\hline Central line & 72 & 57.6 \\
\hline Peripheral line & 53 & 42.4 \\
\hline Age of blood & 15 days & 2 - 36 days \\
\hline \multicolumn{3}{|l|}{ Medications } \\
\hline ACE / ARB inhibitors & 21 & 16.8 \\
\hline Spironolactone & 8 & 6.4 \\
\hline Insulin & 38 & 30.4 \\
\hline Diuretics & 66 & 52.8 \\
\hline \multicolumn{3}{|l|}{ Morbidities } \\
\hline Acidosis $(\mathrm{pH}<7.3)$ & 63 & 50.4 \\
\hline Acute renal failure & 75 & 60 \\
\hline End-stage renal disease & 12 & 9.6 \\
\hline
\end{tabular}

associated complication [5]. Recent research has focused on "stored blood" with growing concern that the age of an RBC unit (even with the current accepted shelf life of 42 days) may affect its safety profile. A large multicenter clinical trial is underway to determine the effects of using standard storage-age RBCs as compared to fresh blood (8 days old) (Age of Blood Evaluation (ABLE) trial, ISRCTN44878718) [1].

Pre-washing of RBCs is an essential practice for reducing potassium load in irradiated PRBCs $[6,7]$. Due to the limited availability of time for preparing washed PRBCs in urgent situations, trauma patients usually receive unwashed RBCs that lead to increased risk for post-transfusion hyperkalemia [7].

Although RBC washing is useful in reducing the potassium load during transfusion, the effectiveness of this preventative technique is unknown in patients with medical co-morbidities such as metabolic acidosis, acute renal failure (ARF), endstage renal disease (ESRD), rhabdomyolysis, hypothermia and shock. In this study, we evaluate the medical morbidity factors and transfusion factors that can contribute to changes in serum potassium levels among critically ill patients.

\section{Aims and objectives}

Primary objective was to assess the statistical significance of the change in serum potassium levels among critically ill patients before and after each unit of PRBC transfusion. Secondary objectives include factors that can potentially cause rise in serum potassium levels in these patients.

\section{Patients and Methods}

\section{Study design}

This is a prospective observational study conducted at Brookdale Hospital from June 2011 to December 2011 that was approved by The Investigational Review Board of Brookdale University Hospital and Medical Center, New York, USA. The study included all consecutive series of patients, greater than 18 years of age, who were admitted to the medical intensive 
Table 2. Univariate and Multivariate Analysis of Factors Affecting $\mathrm{K}^{+}$Value

\begin{tabular}{lllll}
\hline Factors affecting $\mathrm{K}^{+}$value & Univariate & $\mathbf{9 5 \%}$ confidence interval & Multivariate & $\mathbf{9 5 \%}$ confidence interval \\
\hline Change in $\mathrm{K}^{+}$value pre- and post-transfusion & 0.2 & $0.58-1.93$ & 0.3 & $0.31-2.88$ \\
Acidosis & 0.1 & $0.22-1.67$ & 0.2 & $0.62-3.02$ \\
Acute renal failure & 0.64 & $0.28-3.33$ & 0.55 & $0.47-2.17$ \\
End-stage renal disease & 0.52 & $0.26-2.12$ & 0.97 & $0.91-4.92$ \\
No. of transfusions & 0.11 & $0.12-1.83$ & 0.32 & $0.48-2.25$ \\
Age of RBC blood & 0.02 & $0.32-0.91$ & 0.04 & $0.14-0.91$ \\
$\mathrm{~K}^{+}$infusion via central access vs. peripheral access & 0.43 & $0.75-4.17$ & 0.82 & $0.76-3.93$ \\
\hline
\end{tabular}

care unit (MICU) and required blood transfusions. Subjects excluded from the study were MICU patients receiving fresh frozen plasmas, platelets, cryoprecipitates and multiple blood products simultaneously. Patients with ESRD receiving PRBC transfusions during dialysis were also excluded.

We collected data on demographics admitting diagnosis, acid-base disorders and basic metabolic panel prior to each $\mathrm{RBC}$ transfusion. Additional information on date of RBC donation, irradiated RBCs, washed PRBC volume and RBC volume $(\mathrm{mL})$ were collected. We repeated basic metabolic panels $4 \mathrm{~h}$ from the time of initiation of blood transfusion. Adverse events were recorded during transfusion and $4 \mathrm{~h}$ after transfusion. Our criteria for defining transfusion-associated hyperkalemia included laboratory evidence of $\mathrm{K}^{+}$levels $5.5 \mathrm{mEq} / \mathrm{L}$ or above.

Data on factors that can potentially affect serum $\mathrm{K}^{+}$concentration were collected and analyzed using SPSS version 15. In addition to medical morbidities, additional data were collected on use of diuretics, vasodilators (angiotensin converting enzyme inhibitors or angiotensin receptor blockers), spironolactones and potassium supplements in intravenous fluid. The primary outcome was the development of hyperkalemia (plasma potassium level greater than or equal to $5.5 \mathrm{mEq} / \mathrm{L}$ ) after a blood transfusion and to assess the change in serum potassium level. Secondary analysis included correlation between the numbers of factors, which can potentially cause rise in serum potassium levels.

\section{Statistics}

SPSS 15.0 (SPSS, Chicago, IL) was used for all data analyses. The baseline data of patients are assessed using frequency distributions and univariate descriptive statistics including measures of central tendency and dispersion. All variables that could potentially affect changes in potassium levels were included in analysis via Pearson correlation. Finally, variables associated with a $\mathrm{P}$ value of less than 0.05 in univariate analysis were included in the logistic regression model for multivariate analysis. Logistic regression analysis was performed separately to assess factors independently associated with the development of hyperkalemia.

\section{Results}

One hundred twenty-five patients received 160 units of PRBC infusions from June 2011 to December 2011. Table 1 summarizes the characteristics of patient population admitted to MICU and required PRBC transfusion. The median age of the study group was 63 years (range 22 - 92 years). Seventy-one $(57 \%)$ were female and $54(43 \%)$ were male. Indications for blood transfusions include septic shock $(n=61)$, non-ST elevation myocardial infarction (NSTEMI) $(n=25)$, acute blood loss due to gastrointestinal bleeding $(\mathrm{n}=15)$, disseminated intravascular coagulation $(n=9)$ and vaginal bleed $(n=1)$. Fourteen patients received transfusion prior to invasive procedure which includes sigmoidoscopy $(\mathrm{n}=5)$, tracheotomy ( $\mathrm{n}$ $=6)$, liver biopsy $(\mathrm{n}=1)$ and debridement of decubitus ulcers $(\mathrm{n}=2)$.

For data analysis, patient received multiple blood transfusions at a given period, $4 \mathrm{~h} \mathrm{~K}^{+}$level from the initiation of first transfusion or the time of basic metabolic panel drawn after first transfusion was used for analysis. In all cases, basic metabolic panel was drawn immediately after transfusion. However, $\mathrm{K}^{+}$level was also measured at the end of multiple blood transfusion to assess the change in potassium at the given period.

For the primary outcome, the mean change of serum $\mathrm{K}^{+}$ was $3.9 \pm 1.1 \mathrm{mEq} / \mathrm{L}($ mean $\pm \mathrm{SD}$ ) compared to $4.3 \pm 1.2$ $\mathrm{mEq} / \mathrm{L} 4 \mathrm{~h}$ post-transfusion for 160 transfused units of PRBC volume. Four percent of patients $(n=5)$ had post-transfusion hyperkalemia whereas $1.6 \%(n=2)$ developed hypokalemia after transfusion. We noticed higher serum $\mathrm{K}^{+}$value in 73 patients $(58.4 \%)$ compared to 52 patients $(41.6 \%)$. The change of serum potassium was statistically not significant $(\mathrm{P}=0.1)$. Table 2 describes the association of each potential factors and development of hyperkalemia in univariate and multivariate analysis.

We analyzed multiple patient factors that can potentially affect serum $\mathrm{K}^{+}$level like acid-base disorder $(\mathrm{pH}<7.3$ or $>7.5)$ $(\mathrm{P}=0.17), \mathrm{ARF}$ (creatinine 1.3 or above) $(\mathrm{P}=0.61)$, ESRD $(\mathrm{P}$ $=0.58)$ and medications $(\mathrm{P}=0.44)$. Mean age of stored blood defined from the time of donation to the time of administration was found to be 12 days (range: 2 - 36 days). Twelve days older blood was used in $64 \%$ of patients $(n=80)$ during first transfusion compared to 45 patients $(36 \%)$ who received blood of less than 12 days of older. The mean change of serum potassium in patients receiving transfusion $\geq 12$ days old blood was $4.1 \pm 0.4 \mathrm{mEq} / \mathrm{L}$ compared to $4.8 \pm 0.3 \mathrm{mEq} / \mathrm{L}($ mean $\pm \mathrm{SD})$ in patients receiving blood 12 days or less old. Sixty-two patients $(77.5 \%)$ had increased change of serum $\mathrm{K}^{+}$value in patients with $>12$ days old blood transfusion compared to $17.7 \%(\mathrm{n}=$ 


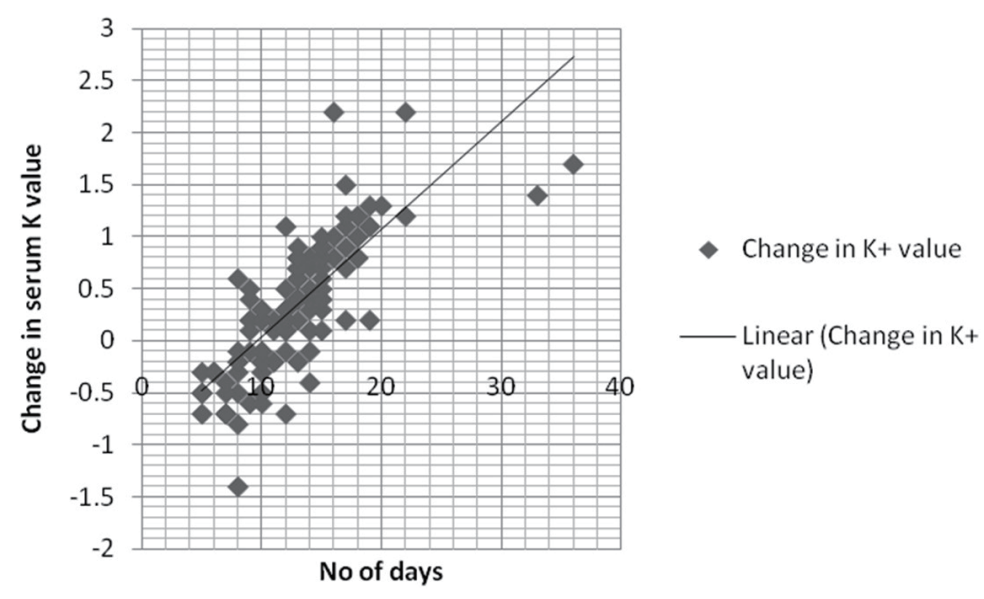

Figure 1. Linear correlation between number of days and serum potassium $\left(\mathrm{K}^{+}\right)$level.

8) patients $(\mathrm{P}=0.02)$. In multivariate logistic regression model, the use of stored PRBC for transfusion was the only factor that affects the increased change in serum $\mathrm{K}^{+}$value $(\mathrm{P}=0.04)$. The change of $\mathrm{K}^{+}$value was independent of acidosis, medications, renal failure, central line versus peripheral line access, and the rate and volume of blood transfusion. Linear regression multivariate analysis for the relationship of transfused stored blood for more than 12 days and the rise in $\mathrm{K}^{+}$level was found to be 0. 889. Figure 1 describes the linear correlation of change in serum potassium and stored blood.

In our study patients, one patient required massive PRBC transfusion due to severe gastrointestinal bleed. Seven units of transfusions were used via pressure bags. However, the PRBC used in the patient were unwashed which could explain the increase in serum potassion from $5.3 \mathrm{mEq} / \mathrm{L}$ to $6.3 \mathrm{mEq} / \mathrm{L}$. One death occurred from cardiac arrest after receiving seven units of PRBCs $(9 \mathrm{mEq} / \mathrm{L})$.

\section{Discussion}

Hyperkalemia is one of the most serious electrolyte disturbances that can lead to lethal cardiac arrhythmias. Smith and colleagues reported 16 patients including 11 adults and five pediatric patients who had cardiac arrests with hyperkalemia during PRBC transfusion with a survival rate of $12.5 \%$ [8]. These findings are reported mostly in surgical literature in settings of large-volume transfusions with other blood products. Therefore, some uncertainty remains to whether use of packed PRBCs is the sole factor which can cause hyperkalemia [4].

Laboratory and observational studies have raised the possibility of prolonged RBC storage that may adversely affect clinical outcomes. In our study, we found that the use of stored PRBC infusions for more than 12 days may lead to elevated $\mathrm{K}^{+}$. However, the magnitude of change in $\mathrm{K}^{+}$by itself is nonsignificant which suggests that present techniques of irradiated washed RBCs to some extent have decreased the complications of hyperkalemia. Stored PRBCs should be used with caution in patients with previously elevated serum $\mathrm{K}^{+}$levels.

In previous reports [3-5], volume of transfusion and rate of PRBCs were factors attributed to rise in $\mathrm{K}^{+}$levels. However, most of these patients received unwashed PRBC transfusions in emergent circumstances that lead to hyperkalemia [3-7]. The citrate-phosphate-dextrose-adenine (CPDA-1) and citrate-phosphate-dextrose (CPD) storage RBCs have shown a 10 -fold rise in $\mathrm{K}^{+}$value through day 1 to day 42 due to decreased RBC survival, increased fragility, decreased viability, and increased deformability of RBCs as well as the release of a number of substances resulting in adverse systemic responses as fever, cellular injury, alterations in regional and global blood flow, and organ dysfunction [9-13].

This is the first study assessing factors that may cause hyperkalemia in critically ill patients. Compared to pediatric and trauma patients, critically ill patients usually receive leuko-reduced and washed PRBCs. Therefore, the change in serum $\mathrm{K}^{+}$ value was never addressed in these patients despite of all these factors. Another important aspect in this study was to measure the $\mathrm{K}^{+}$level immediately after PRBC transfusion. This strategy was employed based on the study by Dani et al [14], where authors have implicated measuring serum $\mathrm{K}^{+}$value immediately after transfusion has provided the accurate estimate of $\mathrm{K}^{+}$ level in their patient population.

In addition to our findings, few clinical trials are testing the hypothesis of increased mortality with use of stored RBCs. The ABLE study is comparing the transfusion of leuko-reduced RBCs stored for 7 days or less (fresh arm) compared to standard-issue RBCs stored, on average, 15 - 20 days (control arm) for 90-day allcause mortality and reduced morbidity in critically ill adults [9].

More recently, $\mathrm{RBC}$ irradiation has shown to damage the $\mathrm{RBC}$ membrane that increases membrane permeability and leakage of potassium into the extracellular space that increases extracellular $\mathrm{K}^{+}$concentration [15]. The washing of RBCs is then performed to reduce $\mathrm{K}^{+}$content, which has been routinely done prior to blood transfusion in medically ill patients in the United States [15]. Therefore, use of washed PRBCs is considered the ideal RBC product in critically ill medical patients. Since washing of PRBCs requires considerable time, patients who require massive transfusions are transfused unwashed PRBCs. As a result, there is a high theoretical risk of hyperkalemia in these patients. 
In the past, majority of these studies were focused on pediatric and adult patients requiring multiple transfusions. Our study demonstrates that stored blood $\mathrm{K}^{+}$load can be attributed to increased serum $\mathrm{K}^{+}$value post-transfusion. This factor was independent on rate and volume of PRBC infusions and medical morbidities. Theoretically, transfusion of larger volumes at increased rate may have the potential to cause a greater predisposition to hyperkalemia. However, in our study, massive transfusion-associated hyperkalemia was noticed only in one patient. The effect of serum potassium levels with large volumes of PRBC transfusion should be closely studied in patients requiring rapid transfusions.

The availability of storage raises the question of how long blood products can and should be stored and how long they are safe and effective and there are no prospective randomized evidence that old RBCs increase mortality despite of theoretical risk $[16,17]$. Few studies are on-going to assess the safety of storage of PRBCs. The ABLE study in critically ill patients and the ARIPI study (NCT00326924) in neonates are assessing the safety of 7 days older storage PRBCs. The "Red Cell Storage Duration and Outcomes in Cardiac Surgery" (NCT00458783) is a single-center RCT studying the safety of 14 days or older PRBCs and "The Red Cell Storage Age Study (NCT00991341) is evaluating the safety of 10 days or older blood. The results of these studies will help to identify the safety cut-off of storage PRBCs [9].

\section{Conclusion}

This is the first prospective study that assesses the change in serum $\mathrm{K}^{+}$levels in critically ill patients. The risk of hyperkalemia is minimal in our patient population due to the use of washed PRBCs. However, our study questions the safety of transfusion of PRBCs stored for greater than 12 days. Determining the optimal duration of RBC storage maximizes safety of transfusion. Future studies should focus on the use of altered storage solution, inclusion of potassium absorption filters during transfusion and cautious use of warmer blood in the hopes of minimizing its clinical consequences through the development of better storage methods [17].

\section{Acknowledgement}

We would like to thank Department of Internal Medicine at Brookdale University Hospital \& Medical Center, New York, USA for their constant support in the clinical research.

\section{Conflict of Interest}

None.

\section{References}

1. Vraets A, Lin Y, Callum JL. Transfusion-associated hy- perkalemia. Transfus Med Rev. 2011;25(3):184-196.

2. Hall TL, Barnes A, Miller JR, Bethencourt DM, Nestor L. Neonatal mortality following transfusion of red cells with high plasma potassium levels. Transfusion. 1993;33(7):606-609.

3. Brown KA, Bissonnette B, McIntyre B. Hyperkalaemia during rapid blood transfusion and hypovolaemic cardiac arrest in children. Can J Anaesth. 1990;37(7):747-754.

4. Sihler KC, Napolitano LM. Complications of massive transfusion. Chest. 2010;137(1):209-220.

5. Rudolph R, Boyd CR. Massive transfusion: complications and their management. South Med J. 1990;83(9):10651070.

6. Knichwitz G, Zahl M, Van Aken H, Semjonow A, Booke M. Intraoperative washing of long-stored packed red blood cells by using an autotransfusion device prevents hyperkalemia. Anesth Analg. 2002;95(2):324-325, table of contents.

7. Chen CH, Hong CL, Kau YC, Lee HL, Chen CK, Shyr $\mathrm{MH}$. Fatal hyperkalemia during rapid and massive blood transfusion in a child undergoing hip surgery--a case report. Acta Anaesthesiol Sin. 1999;37(3):163-166.

8. Smith HM, Farrow SJ, Ackerman JD, Stubbs JR, Sprung J. Cardiac arrests associated with hyperkalemia during red blood cell transfusion: a case series. Anesth Analg. 2008;106(4):1062-1069, table of contents.

9. Lacroix J, Hebert P, Fergusson D, Tinmouth A, Blajchman MA, Callum J, Cook D, et al. The Age of Blood Evaluation (ABLE) randomized controlled trial: study design. Transfus Med Rev. 2011;25(3):197-205.

10. Klein HG, Anstee DJ (Eds.). Mollison's blood transfusion in clinical medicine (11th ed). Blackwell, Malden, MA: Oxford; 2005. p. 687.

11. Sezdi M, Bayik M, Ulgen Y. Storage effects on the ColeCole parameters of erythrocyte suspensions. Physiol Meas. 2006;27(7):623-635.

12. Hornsey VS, MacDonald S, Drummond O, Bethel H, Walker B, Prowse CV. In vitro properties of red cells prepared from half-strength citrate CPD/RAS-2 (Erythro-sol) donations in PL-146 plastic. Transfus Med. 2000;10(1):31-35.

13. Simon GE, Bove JR. The potassium load from blood transfusion. Postgrad Med. 1971;49(6):61-64.

14. Dani C, Perugi S, Benuzzi A, Corsini I, Bertini G, Pratesi S, Rubaltelli FF. Effects of red blood cell transfusions during the first week of life on acid-base, glucose, and electrolytes in preterm neonates. Transfusion. 2008;48(11):2302-2307.

15. Brugnara $\mathrm{C}$, Churchill WH. Effect of irradiation on red cell cation content and transport. Transfusion. 1992;32(3):246-252.

16. Gould S, Cimino MJ, Gerber DR. Packed red blood cell transfusion in the intensive care unit: limitations and consequences. Am J Crit Care. 2007;16(1):39-48; quiz 49.

17. Rusk DM, Eames PM. Modern blood filters do not need to be routinely changed for the majority of red blood cell transfusions in New Zealand. Anaesth Intensive Care. 2010;38(4):685-689. 